Symposiums

\title{
Markedly Induced Expression of LR11 in Atherosclerosis
}

\author{
Hideaki Bujo and Yasushi Saito \\ Second Department of Internal Medicine, Chiba University School of Medicine, Chiba, Japan.
}

\begin{abstract}
Receptors belonging to the low density lipoprotein receptor (LDLR) superfamily play important biological roles in addition to mediating the lipoprotein metabolism. The recent discovery of a novel mosaic LDLR family member by us (Yamazaki H, Bujo H, Kusunoki J, Seimiya K, Kanaki T, Morisaki N, Schneider WJ, and Saito Y J Biol Chem 271: 24761-24768, 1996) and others, which we termed LR11, offers the opportunity to gain new insights into receptor multifunctionality. The expression of a 250-kDa mosaic LDLR family member, which we termed LR11 due to the presence of 11 ligand binding repeats, is markedly induced during the process of atherogenesis in two animal models. The highest induction of LR11 occurs in the intimal smooth muscle cells (SMCs) of atheromatous lesions. In agreement with the correlation of LR11 induction during increased cell proliferation in vivo, cultured SMCs showed a marked increase in LR11 expression in the proliferative phase. Furthermore, such proliferation-dependent expression of LR11 could be observed in a cultured neuroblastoma cell line, which was established to be a suitable in vitro model for proliferation and differentiation. Possible involvement of LR11 in the cellular proliferation sheds new light on the recently proposed novel functions of the LDL receptor gene family in atherosclerosis. J Atheroscler Thromb, $2000 ; 7: 21-25$.
\end{abstract}

Key words : LDLR, Atherosclerosis, Smooth muscle cell, Proliferation

\section{LR11, discovered as a member of the LDLR family with novel mosaic structure}

The discovery of the LDL receptor (LDLR) and its functional and genetic characterization were landmarks in research on lipoprotein transport pathways and led to the molecular delineation of the common hereditary disease, familial hypercholesterolemia. In contrast to the clarity of our understanding of LDLR function, the physiological roles of new LDLR homologues, which have been identified in the past decade and form the ever expanding LDLR gene family (Fig. 1), are far from being established. Their apparently much more diverse functions are indicated by a wide range of possible ligands, which include spent, biologically inactive and/or unwanted plasma carrier complexes and complex proteins, certain toxins, yolk precursors, as well as circulating plasma lipoproteins.

Address for correspondence: Hideaki Bujo, Second Department of Internal Medicine, Chiba University School of Medicine, 1-8-1 Inohana, Chuo-ku, Chiba 260-0856, Japan. E-mail : hbujo@intmed02.m.chiba-u.ac.jp

Received March 10, 2000
The bewildering array of ligands they can bind, at least in vitro, is probably due to their highly variable content of different numbers and combinations of common structural elements.

Some members of the LDLR gene family are suggested to play important roles in accelerated atheroma formation in the arterial wall $(1,2)$. Atherosclerotic lesions are characterized mostly by accumulation in the intima of excessive lipid, and foam cells arise from macrophages migrating through endothelial cells, and from smooth muscle cells (SMCs) in the medial layer (3). Recent extensive histochemical studies have shown that receptors belonging to the LDLR family, as well as scavenger receptors mediating the incorporation of modified lipoproteins such as oxidized low density lipoprotein, show marked induction of their expression during the formation of atherosclerotic lesions (4-8).

We have discovered and molecularly characterized a novel, unusually complex and highly conserved member of the LDLR gene family (9-11). The predominant domain of this type I membrane protein consists of a cluster of eleven LDLR ligand binding repeats; according to our 


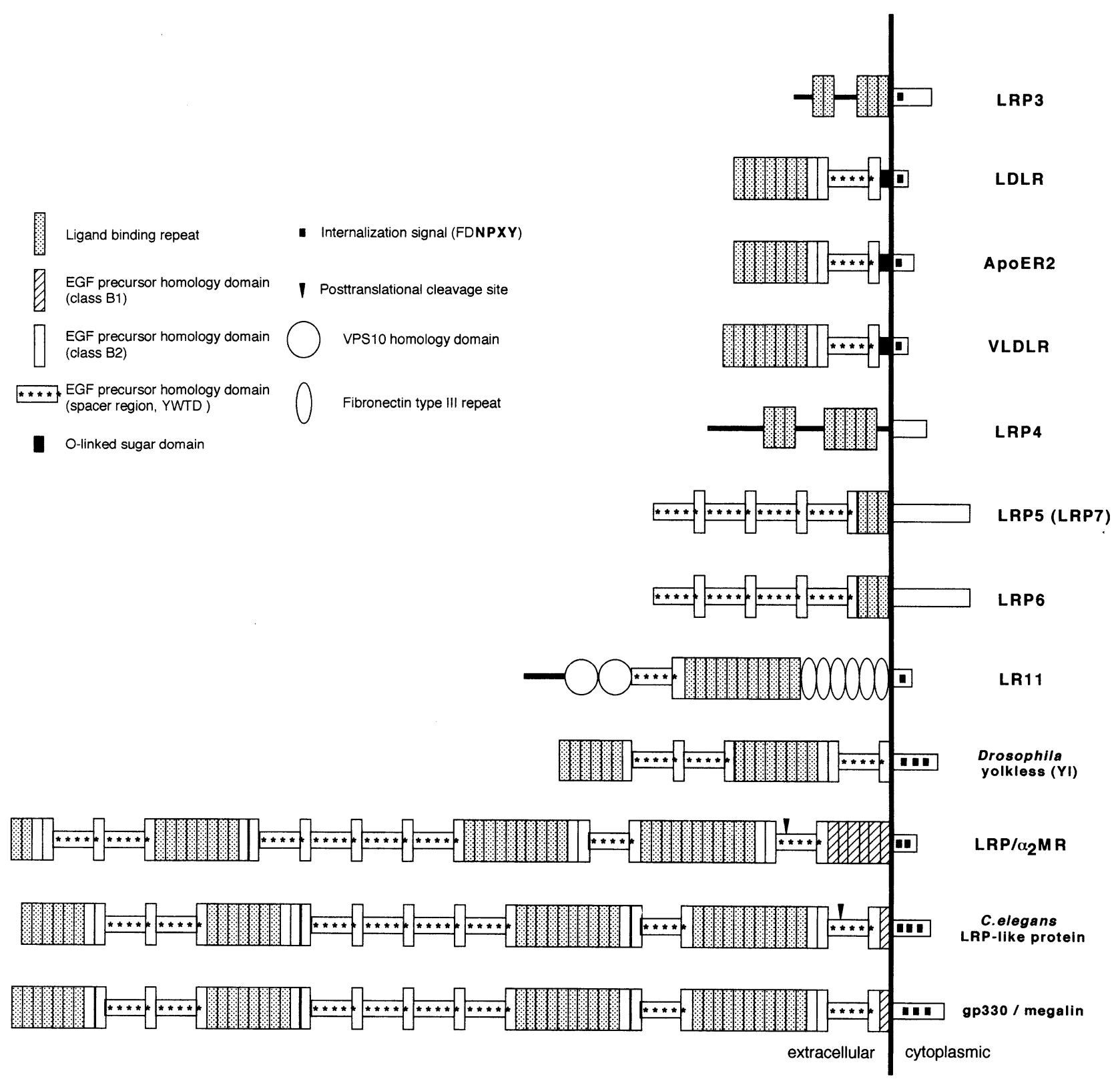

Fig. 1. The LDLR gene family.

The diagram compares the functional domains in the LDL receptor, VLDL receptor, Apo ER2, LRPs-3, 4, 5, 6 and 7, LR11, Drosophila yolkless, LDL receptor-related protein $/ \alpha 2$-macrogloburin receptor (LRP/ $\alpha 2 \mathrm{MR})$, C. Elegans LRP-like protein and gp330/megalin.

preferred nomenclature, we termed the new receptor LR11. Another feature of LR11 is the presence of an extracellular domain homologous to the yeast vacuolar protein sorting receptor, Vps10p ; thus, Gliemann and colleagues named the receptor sorLA-1 (where LA stands for LDLR class A repeats) (12). Another intriguing characteristic of LR11 is the presence of six fibronectin type III repeats following the 11 LDLR binding repeats. The similarity with the repeats in $\mathrm{N}$-CAMs, murine $\mathrm{L} 1$, and their homologues suggests a potential role of LR11 in cell-cell interaction.
Furthermore, the unique expression pattern of the LR11 mRNA in brain and other organs active in morphogenesis during murine development is compatible with roles in neural development and organ formation $(11,13)$.

We have recently studied the regional changes in mRNA and protein levels of LR11 during atheroma formation (14). We have used rabbit and rat arteries as models for experimental atherosclerosis induced by cholesterol feeding and balloon injury, respectively. We also assessed the regulatory features of expression of the mosaic 
receptor in cultured SMCs in vitro. In addition, we studied the LR11 expression in several cell lines, which have been developed to model cellular proliferation and differentiation, in order to clarify the regulatory mechanism of receptor expression.

\section{LR11 expression in atherosclerotic arteries in high-cholesterol diet-fed rabbits}

In order to elucidate the possible pathological roles of LR11 in atheroma formation, we analyzed the expression levels of LR11 mRNA in arteries from rabbits fed an atherogenic diet. Using RT-PCR and a solution hybridization RNase protection assay, LR11 mRNA levels in aortas from rabbits fed a high-cholesterol diet were found to increase 6.8-fold compared to those from normal chow-fed animals. In contrast, LR11 mRNA levels in carotid arteries from rabbits fed a high cholesterol diet increased 1.7-fold compared to those from normal chowfed rabbits. These results strongly suggested increased expression of LR11 mRNA in atherosclerotic arteries.

To examine the localization of LR11 protein in atheromatous lesions, we performed immunohistochemical analysis. In aortic areas without atherosclerotic lesions in the control rabbits, no significant positive labeling for LR11 was seen with the specific antibodies directed against domain IV of the receptor. In aortas from the rabbits fed the high cholesterol diet, clear positive signals for LR11 were detected in the region of intimal thickening.

The LR11 signals in the intima mostly overlapped with those revealed by a cytochemical marker for SMCs, suggesting that the LR11 protein is localized over SMCs in the intima. In addition, LR11 expression, although at lower levels than in the intima, was also detected in medial SMCs close to the intima. These results indicate that the increased levels of LR11 transcripts in atherosclerotic aortas from rabbits fed a high cholesterol diet observed were caused mainly by increased expression of LR11 in intimal SMCs.

\section{LR11 expression in intimal hyperplasia by endothelial denudation}

It has been shown that atheromatous lesions formed by balloon-injury endothelial denudation have higher intimal SMC content than those induced by high cholesterol diet feeding (15). Intimal hyperplasia in experimental vascular remodeling results primarily from an excessive proliferative response of SMCs, which also undergo dedifferentiation and migration. The expression of LR11 transcripts during atheroma formation after balloon injury showed that faint signals were seen after 3 days and a marked increase after 14 days following denudation. Increased mRNA expression of LR11 and of cyclins following denudation suggests that LR11 might be expressed in proliferative intimal cells, such as SMCs.
In situ hybridization analysis of LR11 mRNA using sections of rat carotid arteries obtained 14 days following denudation showed that LR11 mRNA hybridization signals were clearly detected in most regions of intimal hyperplasia, together with the faint signals in medial SMCs, although in control rat carotid areas only faint positive labeling for LR11 mRNA was seen in the medial layer with the antisense probe corresponding to LR11 domains I and II. These results suggested that, as in the rabbit model, LR11 transcripts are elevated in the intimal SMCs in the endothelial denudation rat model.

\section{Regulatory expression of LR11 in cultured SMCs}

Cultured SMCs in quiescence showed low but significant levels of LR11 mRNA, as assessed by the solution hybridization RNase protection assay. Upon culture, LR11 mRNA rose 2.4 fold within one day and increased gradually for 4 days after addition of serum, consistent with being related to cell proliferation activities. The maximum amount of mRNA expression was 21-fold that before adding serum. Thereafter, the expression of LR11 mRNA decreased again; at day 12 , when cell numbers had reached a plateau, the level of LR11 transcript still was 5.6-fold that before serum addition.

Next, in order to examine possible regulatory factors for the receptor in proliferating SMCs, the effects on LR11 expression of medium that had been preconditioned with cultured macrophages (THP-1 cells) were assessed. Northern blot analysis revealed that at $24 \mathrm{hrs}$ after inducing cell proliferation by serum addition, expression of LR11 mRNA was induced 2.2 times above the basal level, consistent with the above results. However, the addition of THP-1-conditioned medium to quiescent SMCs resulted in a 8.6- fold increase in LR11 mRNA when compared to the level in cells that had received 10\% FBS. At the protein levels, LR11 in quiescent cultured SMCs was visualized under our conditions as a $250 \mathrm{kDa}$ protein, as described previously in various tissues (9). The LR11 protein in SMCs was increased slightly $24 \mathrm{~h}$ after addition of $10 \%$ FBS. Significantly, incubation with THP-1-conditioned medium for 24 and $48 \mathrm{hr}$ resulted in 4.4 - and 6.2 fold increases in LR11 protein abundance, respectively, over that obtained by adding $10 \%$ FBS. These effects on LR11 protein expression, together with the results obtained by mRNA analysis, strongly suggested that LR11 expression in cultured SMCs responds to the addition of macrophage-conditioned medium.

\section{Increased expression of LR11 in proliferating neuroblastoma cells}

In order to establish that the induced expression of LR11 mRNA and protein is caused by general molecular machinery accompanied by cellular proliferation, the LR11 protein levels in IMR32 cells were analyzed, because 
IMR32 is known to show two distinct morphological characteristics (proliferated or differentiated), depending on the culture medium. The LR11 protein increased to 5.3 fold at $72 \mathrm{~h}$ in the proliferating cells. The IMR32 cells in differentiation medium showed a 2.9 fold increase within $24 \mathrm{~h}$, thereafter the protein levels decreased to 0.6 fold at $72 \mathrm{~h}$. In the maintenance medium, the LR11 transcripts increased to 4.7 fold at $72 \mathrm{~h}$. In the differentiation medium, IMR32 also increased 1.8 fold within $24 \mathrm{~h}$, and thereafter the levels decreased to 1.1 fold at $72 \mathrm{~h}$. These results suggested that the expression of LR11 protein is dependent on the cellular growth in the neuroblastoma cells, and is markedly induced in the proliferating stage.

\section{The possible roles of LR11 and other members of the LDLR family in atherogenesis}

To date, the only identified ligands of LR11 are the apoE-rich lipoprotein, $\beta$-VLDL (9) and the $39 \mathrm{kDa}$ receptor-associated protein (RAP) (12), in agreement with the notion that all previously described LDLR family members bind both ligands (for reviews, see ref. 2). Recently, Hermans-Borgmeyer et al. reported that the putative LR11 homologue of the coelenterate hydra is a specific receptor for the neuropeptide "head activator", which stimulates head-specific growth and differentiation processes in this invertebrate (13). If this analogy holds, the peptide's function in mammals might be the stimulation of proliferation of neural precursor cells, stabilization of nerve cell survival, and enhancement of neural outgrowth. At present, in the absence of a transgenic animal model, we cannot determine which ligand(s) may be the true physiological partner(s) of LR11. However, the structural feature of homology with neural adhesion molecules possibly suggests that at least part of LR11's function might be in the process of cell-cell interaction and differentiation of various cells, similar to the roles of N-CAM and L1 (9). On the other hand, the homology to the putative ligand binding regions of a yeast carboxypeptidase $\mathrm{Y}$ sorting receptor is compatible with LR11 being involved in the action of certain proteases reported to be important for cell growth and migration. In agreement with these features of LR11, transcripts have been detected during periods of active morphogenesis, suggesting a role for this receptor in the proliferation that occurs before differentiation of many embryonic cells and tissues $(11,13)$.

So far the physiological significance, if any, of the expression of LDLR gene family members in arterial walls has not been fully elucidated. Certain LDLR relatives are expressed in the arterial wall, implying possible roles in the progression of atherosclerotic plaques (Table 1). For instance, LRP has been demonstrated in lesion macrophages and SMCs $(4,8)$, and VLDLR/LR8 in endothelial cells, macrophages and SMCs (5-8). In contrast, the LDLR is not abundant in arterial walls $(4,8)$. LRP has been demonstrated to be involved in the migration and proliferation of various cells related to atherosclerosis and cancer invasion. Embryonic fibroblasts genetically deficient in LRP show increased activity of the urokinase receptor system and accelerated migration on vitronectin (16).

The expression pattern of LR11 in the arterial walls suggests that it may be involved in smooth muscle cell (patho)biology, such as migration and proliferation into the intima from the media. The characteristic expression during rapid proliferation and induction by macrophageconditioned medium suggests roles of LR11 in the growth and differentiation of vascular SMCs, as well as certain functions in other cells during embryogenesis $(11,13)$. Modulation of the SMC phenotype accompanying the migration of medial SMCs through the internal elastic lamina into the intima has been proposed by several observers $(17,18)$. The modulation process can be triggered by intimal macrophages, which degrade the heparan sulfate proteoglycan component of the SMC basal lamina via membrane-bound or secreted proteases and endoglycosidase $(19,20)$. Macrophages also produce a number of growth factors for SMCs such as plateletderived growth factor (PDGF) (21). The enhanced expression of LR11 by macrophage-conditioned medium in vitro possibly reflects its induction in rabbit SMCs of atherosclerotic arteries ; the expression of LR11 might be regulated by factors secreted from the macrophages in the hyperplastic intima. The elucidation of the expression mechanism of the novel mosaic receptor, LR11, in SMCs and other proliferating cells could identify the functional

Table 1.

\begin{tabular}{|c|c|c|c|}
\hline & \multirow{2}{*}{ Normal arteries } & Atheroma & \multirow[b]{2}{*}{ Regulatory factors } \\
\hline & & Early Advanced & \\
\hline LDL receptor & - & - & $\begin{array}{l}\text { Macrophage differentiation (P) } \\
\text { TGF- } \beta(\mathrm{P}) \\
\text { Intracellular cholesterol }(\mathrm{N})\end{array}$ \\
\hline VLDL receptor & $\stackrel{+}{+}$ & (EC, Macrophage, $\stackrel{+}{+}$ SMC) & GC-MCSF (P) \\
\hline LRP $/ \alpha 2 \mathrm{MR}$ & $\stackrel{\#}{(\mathrm{SMC})}$ & $\stackrel{\#}{\text { (Macrophage, }} \stackrel{\#}{H C})$ & $\begin{array}{l}\text { Macrophage differentiation }(P) \\
\text { MCSF(P) } \\
\text { Lipopolysaccharides }(\mathrm{N})\end{array}$ \\
\hline LR11 & - & (SMC) & $\begin{array}{l}\text { Cellular proliferation }(\mathrm{P}) \\
\text { Macrophage-conditioned medium }(\mathrm{P})\end{array}$ \\
\hline
\end{tabular}


significance of the LDL receptor gene family, which has recently been strongly suggested to be essential for neuronal organization using VLDLR and apoER2 double knock out mice (14).

Acknowledgements: We would like to thank Drs. T. Kanaki, S. Hirayama, I. Ishii and N. Morisaki (Chiba University) for their great contributions.

\section{References}

(1) Ylä-Herttuala S: Expression of lipoprotein receptors and related molecules in atherosclerotic lesions. Cur Opin Lipidol, 7 : 292-297, 1996

(2) Yamamoto $\mathrm{T}$ and Bujo $\mathrm{H}$ : Close encounters with apolipoprotein E receptors. Cur Opin Lipidol, 7 : 298302, 1996

(3) Ross R: The pathogenesis of atherosclerosis: a perspective for the 1990s. Nature, 362: 801-809, 1993

(4) Luoma JS, Hiltunen TP, Särkioja T, Moestrup SK, Gliemann J, Kodama T, Nikkari T, and Ylä-Herttuala S : Expression of alpha2-macroglobulin receptor/low density lipoprotein receptor-related protein and scavenger receptor in human atherosclerotic lesions. J Clin Invest, 93: 2014-2021, 1994

(5) Wyne KL, Pathak PK, Seabra MC, and Hobbs HH: Expression of the VLDL receptor in endothelial cells. Arterioscler Thromb Vasc Biol, 16: 407-415, 1996

(6) Nakazato K, Ishibashi T, Shindo J, Shiomi M, and Maruyama $Y$ : Expression of very low density lipoprotein receptor mRNA in rabbit atherosclerotic lesions. Am $\mathrm{J}$ Pathol, 149: 1831-1838, 1996

(7) Multhaupt HAB, Gåfvals ME, Kariko K, Jin H, ArenasElliot C, Goldman BI, Straus JF III, Angelin B, Warhol MJ, and McCrae KR: Expression of very low density lipoprotein receptor in the vascular wall : analysis of human tissues by in situ hybridization and immunochemistry. Am J Pathol, 148: 1985-1997, 1996

(8) Hiltunen TP, Luoma JS, Nikkari T, and Ylä-Herttuala S : Expression of LDL receptor, VLDL receptor, LDL receptor-related protein, and scavenger receptor in rabbit atherosclerotic lesions. Marked induction of scavenger receptor and VLDL receptor expression during lesion development. Circulation, 97 : 1079-1086, 1998

(9) Yamazaki H, Bujo H, Kusunoki J, Seimiya K, Kanaki T, Morisaki N, Schneider WJ, and Saito Y: Elements of neural adhesion molecules and a yeast vacuolar protein sorting receptor are present in a novel mammalian low density lipoprotein receptor family member. J Biol Chem, 271: 24761-24768, 1996

(10) Moerwald S, Yamazaki H, Bujo H, Kusunoki J, Kanaki T, Seimiya K, Morisaki N, Nimpf J, Schneider WJ, and Saito $Y$ : A novel mosaic protein containing LDL receptor elements is highly conserved in humans and chickens.
Arterioscler Thromb Vasc Biol, 17 : 996-1002, 1997

(11) Kanaki T, Bujo H, Hirayama S, Tanaka K, Yamazaki H, Seimiya K, Morisaki N, Schneider WJ, and Saito Y. Developmental regulation of LR11 expression in murine brain. DNA Cell Biol, 17 : 647-657, 1998

(12) Jacobsen L, Madsen P, Moestrup SK, Lund AH, Tommerup N, Nykjaer A, Sottrup-Jensen L, Gliemann J, and Petersen CM: Molecular characterization of a novel human hybrid-type receptor that binds the $\alpha_{2}$-macroglobulin receptor associated protein (RAP). J Biol Chem, 271: 31379-31383, 1996

(13) Hermans-Borgmeyer I, Hampe W, Schinke B, Methner A, Nykjaer A, Suesens U, Fenger U, Herbarth B, and Schaller $\mathrm{HC}$ : Unique expression pattern of a novel mosaic receptor in the developing cerebral cortex. Mech Dev, 70: 65-76, 1998

(14) Kanaki T, Bujo H, Hirayama S, Ishii I, Morisaki N, Schneider WJ, and Saito $Y$ : Expression of LR11, a mosaic LDL receptor family member, is markedly increased in atherosclerotic lesions. Arterioscler Thromb Vasc Biol, 19 : 2687-2695, 1999

(15) Wei GL, Krasinski K, Kearney M, Isner JM, Walsh K, and Andrés V. Temporally and spatially coordinated expression of cell cycle regulatory factors after angioplasty. Circ Res, 80: 418-426, 1997

(16) Weaver AM, Hussaini IM, Mazar A, Henkin J, and Gonias $\mathrm{SL}$ : Embryonic fibroblasts that are genetically deficient in low density lipoprotein receptor-related protein demonstrate increased activity of the urokinase receptor system and accelerated migration on vitronectin. J Biol Chem, 272: 14372-14379, 1997

(17) Orlandi O, Ehrlich HP, Ropraz P, Spagnoli LG, and Gabbiani $G$ : Rat aortic smooth muscle cells isolated from different layers and at different times after endothelial denudation show distinct biological features in vitro. Arterioscler Thromb, 14 : 982-989, 1994

(18) Bochaton-Piallat ML, Gabbiani G, and Pepper MS: Plasminogen activator expression in rat arterial smooth muscle cells depends on their phenotype and is modulated by cytokines. Circ Res, 82: 1086-1093, 1998

(19) Rennick RE, Campbell JH, and Campbell GR: Vascular smooth muscle cell phenotype and growth behaviour can be influenced by macrophages in vitro. Arteriosclerosis, 71: 35-43, 1988

(20) Campbell JH, Rennick RE, Kalevitch SG, and Campbell GR: Heparan sulfate-degrading enzymes induce modulation of smooth muscle phenotype. Exp Cell Res, 200 : 156-167, 1992

(21) Raines EW and Ross R: Multiple growth factors are associated with lesions of atherosclerosis: specificity or redundancy? BioEssays, $18:$ 271-282, 1995

(22) Trommsdorff M, Gotthardt M, Hiesberger T, Shelton L, Stockinger W, Nimpf J, Hammer RE, Richardson JA, and Herz $\mathrm{J}$ : Reeler/Disabled-like disruption of neuronal migration in knockout mice lacking the VLDL receptor and ApoE receptor 2. Cell, $97: 689-701,1999$ 